Background Streamlining the timing of treatments in CF is important to optimise adherence whilst ensuring efficacy. The optimal timing of HTS and ACTs is unknown.

Objectives This study hypothesised that ACTs after HTS would be more effective than ACTs during HTS as measured by lung clearance index (LCI). FEV1, sputum weight and patient perceptions were also compared.

Methods Adults with CF providing written informed consent were randomised (between days 10-14 of intravenous antibiotic course during a pulmonary exacerbation) to a crossover trial of ACTs after HTS inhalation or ACTs during HTS inhalation on alternate days. ACT treatment consisted of 10 cycles of active cycle of breathing technique using an Acapella ${ }^{\circledR}$. The physiotherapist collecting the outcome measures was blinded. Patients completed a Multiple Breath Washout (MBW) test to obtain LCI and spirometry at baseline and 90 mins post treatment. Sputum collection during 90 mins, ease of clearance and satisfaction with treatment was also recorded. Wilcoxon test was used and $\mathrm{p}<0.05$ was considered significant.

Results Fourteen subjects were recruited and 13 completed the study (mean [SD] age 33 [12], FEV1\% predicted 51 [22], LCI (no. turnovers) 14 [4]). Comparing the 2 treatments (ACT after HTS vs ACT during HTS), the change from baseline to 90 mins post treatment in LCI $(\mathrm{p}=0.71)$ and $\mathrm{FEV} 1 \%$ predicted $(\mathrm{p}=0.97)$ was not significant. There was also no difference in sputum weight expectorated ( $\mathrm{p}=0.17)$, patient perceived ease of clearance $(p=0.33)$ or satisfaction $(p=0.28)$. The time taken for ACT during HTS was significantly shorter $(p=0.001)$.

Conclusions In this small study, ACTs after HTS was no more effective than ACTs during HTS.

\section{P96 PHYSIOTHERAPY MANAGEMENT OF ADULT PATIENTS WITH CYSTIC FIBROSIS ON INTENSIVE CARE UNITS (ICU) - A SURVEY OF UK PHYSIOTHERAPISTS}

F Cathcart, H Parrott, A Jones, N Simmonds. Adult Cystic Fibrosis Centre, Royal Brompton Hopsital, London, UK

\subsection{6/thoraxjnl-2016-209333.239}

Introduction and objectives Currently no guidelines or data exist on the physiotherapy management of adults with CF on the ICU. The aim was to explore the views and experiences of the specialist physiotherapists (SPs) managing adults with CF admitted to an ICU.

Methods An online survey was designed which included questions (open and closed) on staff confidence, communication, learning needs, extracorporeal membrane oxygenation (ECMO) and end of life care. The survey was sent to CF, ICU and transplant SPs across the UK.

Results 42 SPs responded (74\% response rate), 52\% (n = 22) adult CF, 40\% ( $\mathrm{n}=17)$ ICU and 7\% $(\mathrm{n}=3)$ transplant specialists. $73 \%(\mathrm{n}=30)$ had been specialists in their area for $>5$ years. $27 \%(\mathrm{n}=11)$ reported no CF admissions to their ICU in the last year, only $24 \%(n=10)$ had $>3$ in the last year. Physiotherapy care was shared between the ICU and CF SPs teams in $43 \%$ $(\mathrm{n}=18)$ of respondents. 90\% $(\mathrm{n}=37)$ felt this joint working was essential to optimise patient care. On a confidence scale of 1 - $10(1=$ low, 10 = high) the median (IQR) confidence score of SPs to manage patients with CF on ICU was 7 (7-9).

43\% ( $n=17)$ had experienced pre transplant patients with $\mathrm{CF}$ being invasively ventilated and reported that the challenges included airway clearance, weaning, inhalation therapy and nursing staff education. Of the 17 respondents who worked in ECMO centres, $57 \%(n=12)$ had never had a patient with CF on ECMO and 90\% had no physiotherapy ECMO protocol. Reported challenges were mobilisation, chest clearance, inhalation therapy and palliative care.

Of respondents 50\% $(\mathrm{n}=21)$ had managed a patient with CF who died on ICU. 79\% $(n=33)$ of respondents wanted more education on managing patients with CF on ICU with comments around, joint training, national guidelines being developed and the importance of collaborative care.

Conclusions The number of adults with CF admitted to ICU remains low nationally however SPs need to maintain competence and confidence in managing these complex patients. National, expert consensus guideline development, including a physiotherapy ECMO protocol would assist in ensuring equitable quality care in this setting.

\section{P97 GASTRO-OESOPHAGEAL REFLUX IN CYSTIC FIBROSIS}

1,2RW Lord, ' 1 JS Pearson, ' $P J$ Barry, ${ }^{1} \mathrm{PJ}$ Whorwell, ${ }^{1} \mathrm{RB}$ Jones, ${ }^{3} \mathrm{P}$ McNamara, ${ }^{4} \mathrm{R}$ Beynon, ${ }^{1,2}$ JA Smith, ${ }^{1}$ AM Jones. ${ }^{1}$ University Hospital South Manchester, Manchester, UK; ${ }^{2}$ University of Manchester, Manchester, UK; ${ }^{3}$ Alder Hey Hospital, Liverpool, UK; ${ }^{4}$ University of Liverpool, Liverpool, UK

\subsection{6/thoraxjnl-2016-209333.240}

Background Initial small studies using combined $\mathrm{pH}$ and impedance ( $\mathrm{pH}-\mathrm{MII})$ have suggested increased gastro-oesophageal reflux (GOR) in adult cystic fibrosis (CF) patients. Reflux episodes frequently reach the proximal oesophagus and occur whilst supine, which may predispose to micro-aspiration.

Aims To investigate the relationship between GOR and CF lung disease.

Methods We conducted a prospective observational study in stable adult CF patients undergoing 24 hour pH-MII. Reflux symptoms (using validated RESQ-7 questionnaire) and spirometry were also recorded.

Results 10/12 subjects recruited completed pH-MII the study (mean age 28.5 years, mean FEV1 48.8\% predicted, 100\% male). An increased number of reflux episodes per 24 hours were noted in $80 \%$ of participants (median 104.1, IQR 78.6 -164.4, normal range $<75$ episodes), with increased 'high-risk' proximal reflux or supine reflux noted in $60 \%$ (Table 1). In this small preliminary patient sample there were no significant correlations between FEV1\% and total, proximal or supine reflux episodes, or RESQ7 heartburn scores and any reflux measure.

$7 / 10$ subjects completed the study on a PPI and had data available for acid reflux $(\mathrm{pH}<4)$ parameters. $4 / 7$ had an abnormal acid exposure despite standard dose PPI therapy. 5/7 had prolonged acid reflux events; the longest was 66.5 minutes (see Table 1).

Conclusions In this preliminary data set, CF patients appear to have a pattern of GOR that puts them at risk of reflux microaspiration and have significant acid reflux despite antacid therapy. Reflux symptoms and spirometry were not related to reflux parameters.

The clinical implications of these findings are that standard PPI dosing maybe insufficient in CF patients to control acid reflux. In addition silent reflux aspiration maybe occurring and as such could be a potential area for future therapies. 